\title{
Non-Perturbative Solutions of String Theory in Gravitational Backgrounds
}

\author{
Octávio A. Mattos \\ King's College London, Department of Mathematics \\ The Strand, London WC2R 2LS - U.K. \\ Victor O. Rivellest \\ Instituto de Física, Universidade de São Paulo \\ P.O. Box 20516, 01498-970 - São Paulo - SP - Brazil
}

\begin{abstract}
We study bosonic string theory in a gravitational background. We show that either left-movers or right-movers are the only background independent nonperturbative solutions of the field equations for an arbitrary static metric. They are stable and have a conserved topological charge being therefore topological solitons. The action vanishes for these solutions and hence they provide the dominating contribution in a path integral quantization.
\end{abstract}

11.17.+Y, 11.10.Lm

Typeset Using REVTEX 
The study of non-perturbative solutions in classical field theories has shed light in many features of the corresponding quantum theory since in general these features cannot be reached by perturbation theory. For instance, topological solitons indicate the existence of hidden sectors of the Hilbert space while the presence of instantons indicates vacuum tunnelling, showing, in both cases, that a rich quantum structure is available. Since there is no manageable field theory for strings the only way to study the interaction of strings is through perturbation theory. Therefore any non-perturbative solution of string theory may give some clues about the symmetries or the geometrical foundations of a string field theory.

Another aim of studying strings in backgrounds consists in finding a (low energy) phenomenologicaly acceptable string vacuum or consistent compactifications. However, as is well known, strings do not propagate in arbitrary backgrounds if conformal symmetry is to be kept at the quantum level [1].

Since we find background independent solutions conformal invariance can be achieved by choosing an appropriate background. Plane waves backgrounds [2], of which shock waves backgrounds [3] are a particular case, as well as group manifolds [4] are examples of backgrounds which keep conformal invariance at the quantum level. Recently, a class of spacetime metric has been proved finite in the presence of the dilaton field [5].

In this paper we study non-perturbative solutions of the bosonic string theory in a gravitational background field. We show that closed string left-movers or right-movers are background independent solutions. However, not all coordinates can be taken as rightor left-movers since in this case the gauge freedom cannot be fixed completely. In the light-cone gauge (LCG) we find that only left- or only right-movers are solutions for static, but otherwise arbitrary backgrounds, and that these backgrounds can be interpreted as a comoving frame for the string. When both movers are present the space-time metric split up in blocks with two of them being still completely arbitrary.

In fact, all coordinates being either left- or right-movers corresponds to the only background independent solution. This solution is stable under small perturbations. Finally we show that the solutions having only the string winding mode can be interpreted as soli- 
tons and a quantum soliton operator can be built. Since the action vanishes for left- or right-movers they give the dominating contribution to the path integral.

The string action in an arbitrary $D$ dimensional gravitational background $G_{\mu \nu}(\mu, \nu=$ $0, \ldots, D-1)$ is given by

$$
S=\frac{1}{2 \pi \alpha^{\prime}} \int d \sigma d \tau \sqrt{g} g^{\alpha \beta} G_{\mu \nu}(X) \partial_{\alpha} X^{\mu} \partial_{\beta} X^{\nu},
$$

where $g_{\alpha \beta}(\alpha, \beta=0,1)$ is the world-sheet metric. The classical equations of motion and constraints are, respectively,

$$
\begin{array}{r}
\square^{2} X^{\mu}+\Gamma_{\nu \lambda}^{\mu} g^{\alpha \beta} \partial_{\alpha} X^{\nu} \partial_{\beta} X^{\lambda}=0, \\
T_{\alpha \beta}=G_{\mu \nu}\left(\partial_{\alpha} X^{\mu} \partial_{\beta} X^{\nu}-\frac{1}{2} g_{\alpha \beta} g^{\alpha^{\prime} \beta^{\prime}} \partial_{\alpha^{\prime}} X^{\mu} \partial_{\beta^{\prime}} X^{\nu}\right)=0,
\end{array}
$$

where $\Gamma_{\mu \nu}^{\lambda}$ is the Christoffel symbol for the metric $G_{\mu \nu}$. In the conformal gauge, (2) and (3) reduce to

$$
\begin{gathered}
\hat{\partial}_{+} \hat{\partial}_{-} X^{\mu}+\Gamma_{\nu \lambda}^{\mu} \hat{\partial}_{+} X^{\nu} \hat{\partial}_{-} X^{\lambda}=0 \\
T_{ \pm \pm}=G_{\mu \nu} \hat{\partial}_{ \pm} X^{\mu} \hat{\partial}_{ \pm} X^{\nu}=0
\end{gathered}
$$

where $\hat{\partial}_{ \pm} \equiv\left(\partial_{\tau} \pm \partial_{\sigma}\right)$.

As it is easily seen, left- or right-movers, satisfying $\hat{\partial}_{+} X^{\mu}=0$ or $\hat{\partial}_{-} X^{\mu}=0$ respectively, are background independent solutions of the field equations (4). The Euclidean version of these solutions have been recognized as instantons in two- dimensional non-linear sigma models for target spaces with non-trivial second homotopy group [6]. In our case, however, there are two more equations to be satisfied namely, the constraints (5). As will be shown, they prove to be very strong, restricting the allowed background space-times.

In two-dimensional Minkowsky space-time, fields satisfying either of the conditions $\hat{\partial}_{+} X^{\mu}=0$ or $\hat{\partial}_{-} X^{\mu}=0$, are known as chiral bosons. They have been studied and their quantization performed in several schemes [7]. In the heterotic string theory, left-mover 
target space coordinates generate the internal symmetry group through compactification on a flat torus [8], whereas in the context of non-linear sigma models, chiral bosonic fields the so called leftons and rightons - have also been considered [9]. We shall denote string coordinates of only a mover type (either left or right) as being 'chiral'.

As it is well known the gauge $g_{\alpha \beta}=\eta_{\alpha \beta}$ still leaves a combination of reparametrization invariance and Weyl scaling to be fixed. This freedom enables us to make a further gauge choice and the light-cone gauge $X^{+} \equiv \frac{1}{\sqrt{2}}\left(X^{0}+X^{D-1}\right)=\left(X_{0}^{+}+P^{+} \tau\right)$ is taken in order to fix this residual invariance [10]. In this gauge, however, the coordinate $X^{+}$is no longer chiral. We could therefore ask whether it is possible to obtain a solution with all coordinates being chiral with a gauge choice such as $X^{+}=X_{0}^{+}+P^{+} \sigma^{+}$(where $\sigma^{ \pm}=\sigma \pm \tau$ ). Nevertheless, this gauge does not completely fix the residual gauge freedom left over by the conformal gauge namely, $\sigma^{+} \rightarrow \tilde{\sigma}^{+}\left(\sigma^{+}\right), \sigma^{-} \rightarrow \tilde{\sigma}^{-}\left(\sigma^{-}\right)$, as can be easily checked.

We now turn to study the backgrounds where not all coordinates are chiral. In order to fix the residual gauge invariance we choose the LCG with the non-chiral coordinate $X^{+}$. The LCG choice will affect the equations of motion (田) where all terms containing $X^{+}$will spoil the possibility of a chiral solution for the remaining coordinates. Starting with a metric such that $G_{++}=G_{--}=0$, which partially sets the coordinate system [1], we can require that these various terms containing $X^{+}$vanish. For right-movers this leads to the equations

$$
\begin{aligned}
2 P^{+} \partial_{+} G_{+-}+\left(\partial_{+} G_{-i}\right. & \left.+\partial_{i} G_{+-}-\partial_{-} G_{+i}\right) \hat{\partial}_{-} X^{i}=0 \\
2 P^{+} \partial_{+} G_{+i}+\left(\partial_{+} G_{-i}\right. & \left.+\partial_{-} G_{+i}-\partial_{i} G_{+-}\right) \hat{\partial}_{-} X^{-} \\
& +\left(\partial_{+} G_{i j}+\partial_{[j} G_{+i]}\right) \hat{\partial}_{-} X^{j}=0
\end{aligned}
$$

where $\partial_{ \pm}$refers to space-time derivatives (while $\hat{\partial}_{ \pm}$refers to world-sheet derivatives). The constraints (5) then imply,

$$
\begin{gathered}
2 P^{+} G_{+-} \hat{\partial}_{-} X^{-}+2 P^{+} G_{+i} \hat{\partial}_{-} X^{i} \\
+2 G_{-i} \hat{\partial}_{-} X^{-} \hat{\partial}_{-} X^{i}+G_{i j} \hat{\partial}_{-} X^{i} \hat{\partial}_{-} X^{j}=0 .
\end{gathered}
$$


Now we must solve () for the various components of the metric as functions of $X^{+}, X^{-}$ and $X^{i}$. It turns out that these equations have a solution for a static (i.e, $X^{+}$independent) background,

$$
\begin{gathered}
G_{i j}=g_{i j}, \quad G_{+i}=\partial_{i} h, \\
G_{+-}=\partial_{-} h, \quad G_{-i}=g_{-i},
\end{gathered}
$$

where $g_{i j}, g_{-i}$ and $h$ are functions of $X^{-}$and $X^{i}$ only. Notice that, apart from being static, (7) corresponds to a choice of a coordinate frame, as the following counting of its independent components shows. The spatial metric $g_{i j}$ has $\frac{1}{2}(D-2)(D-1)$ independent components while $g_{-i}$ has $D-2$ and $h$, one. Altogether they give the $\frac{1}{2} D(D-1)$ independent components of a D-dimensional metric after a choice of a coordinate frame is made.

The static character of the solution (7) stems from the fact that the chiral coordinates $X^{-}$and $X^{i}$ depend symmetrically on the world-sheet parameters $\sigma$ and $\tau$ while, in the LCG, this symmetry is absent in $X^{+}$. Therefore, an equation of motion for the string coordinates cannot in principle be satisfied once the background depends on $X^{+}$, but must be static. It can also be understood with the following argument. If we start with a flat space-time where there is a closed string with both left- and right-movers, then we could go over to a curved background where only one of the movers remain, according to our solution. As this cannot happen, it means that it is not possible to curve the flat space. Therefore we must have a static background, or at least a background with a time dependence that can never reach flat space. If we insist in curving the background we would find a solution with both movers mixed up non-linearly in order to satisfy the equations of motion (4) and the constraints (5).

An important feature of our solution is that the center-of-mass momentum is always equal (up to a sign) to its winding number, as can be seen, for instance, from the right-mover

$X_{R}^{i}(\sigma, \tau)=x_{R}^{i}+\left(\frac{P^{i}}{2}-L^{i}\right)(\tau-\sigma)+\frac{i}{2} \sum_{n \neq 0} \frac{1}{n} \tilde{\alpha}_{n} e^{-2 i n(\tau-\sigma)}$

where $x_{R}^{i}$ is the zero-mode and, $P^{i}$ and $L^{i}$ are the momenta and winding modes of the 
associated non-chiral string which now appear combined to give what is both the momenta and the winding number of the chiral string. This makes the string to slip over itself in such a way that its center-of-mass is always at rest. In fact, the class of metrics (7) corresponds to a comoving frame for the string.

For a relativistic point particle the comoving frame is defined by 12

$$
d s^{2}=d t^{2}-G_{i j}(X, t) d X^{i} d X^{j}
$$

Once we go to the proper-time gauge $X^{0}=P^{0} \tau$ (it plays a role similar to the conformal and the LCG for the string), the equations of motion for the point particle take the form

$$
\ddot{X}^{i}+G^{i k} \dot{G}_{k j} \dot{X}^{j}-\frac{1}{2} G^{i j} \partial_{j} G_{k l} \dot{X}^{k} \dot{X}^{l}=0
$$

and the point particle constraint, $P^{2}=m^{2}$, becomes,

$$
\left(P^{0}\right)^{2}-G_{i j} \dot{X}^{i} \dot{X}^{j}=m^{2}
$$

where $m$ is the particle rest mass. The only background independent solution to these equations is $\dot{X}^{i}=0$. To see this take the partial derivative of the constraint (10b),

$$
\left(\partial_{k} G_{i j}\right) \dot{X}^{i} \dot{X}^{j}=0
$$

Thus, (10a) gives, $\partial_{\tau}\left(G_{i j} \dot{X}^{j}\right)=0$, or $\dot{X}^{t}=G^{j k} C_{k}$, for a constant $C_{k}$. Substituting in (10b), gives $C_{k} C_{l} G^{k l}=$ cte. Since $G_{k l}$ is arbitrary, this identity can only be satisfied if $C_{k}=0$, or $\dot{X}^{i}=0$. The equation of motion for $X^{0}$ (namely $\left.\dot{X}^{i} \dot{X}^{j} \partial_{0} G_{i j}=0\right)$ is consistent with (10b) and was not directly used in this derivation.

For the string, it can be easily demonstrated, with a metric of the form

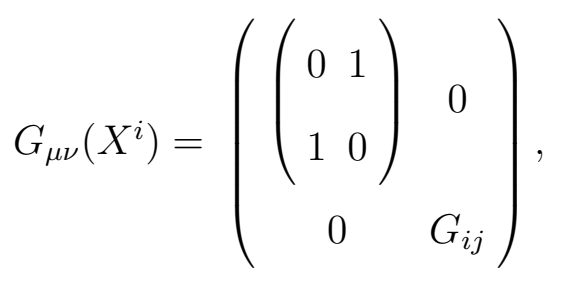

that either left- or right-movers are the unique background independent solutions. 
The equation of motion for $X^{+}$gives $\partial_{-} G_{i j}=0$, while the equation of motion for $X^{-}$(which is the same as for the flat case), for the $X^{i}$, and the constraints are, respectively,

$$
\begin{gathered}
\hat{\partial}_{+} \hat{\partial}_{-} X^{-}=0 \\
\hat{\partial}_{+} \hat{\partial}_{-} X^{i}+\frac{1}{2} G^{i l}\left(\partial_{j} G_{k l}+\partial_{k} G_{j l}-\partial_{l} G_{j k}\right) \hat{\partial}_{+} X^{i} \hat{\partial}_{-} X^{k}=0 \\
2 P_{+} \hat{\partial}_{ \pm} X^{-}=G_{i j} \hat{\partial}_{ \pm} X^{i} \hat{\partial}_{ \pm} X^{j} .
\end{gathered}
$$

From (13a) and (13a) one has

$$
G_{i j} \hat{\partial}_{ \pm} X^{i} \hat{\partial}_{ \pm} X^{j}=F\left(\operatorname{sigma}^{ \pm}\right)
$$

This last equation allow us in fact to have both movers. To take this into account let us split the coordinates $X^{i}$ into left-movers coordinates $X^{A},\left(\hat{\partial}_{-} X^{A}=0, A=1, \ldots, D^{\prime}-1\right)$ and right movers $X^{a},\left(\hat{\partial}_{+} X^{a}=0, a=D^{\prime}, \ldots, D-2\right)$. Without loss of generality, the components of the metric $G_{i j}$ can also be split into $G_{a b}, G_{A B}$ and $G_{a B}$. Then from (14) we obtain $G_{A B}$ as an arbitrary function of $X^{A}$ and similarly for $G_{a b}$, while from (13b) one gets,

$$
\begin{array}{cc}
\left(\partial_{B} G_{A b}-\partial_{A} G_{B b}\right) \hat{\partial}_{-} X^{b} \hat{\partial}_{+} X^{B}=0 & \text { for } \mathrm{i}=\mathrm{A} \\
\left(\partial_{b} G_{a B}-\partial_{a} G_{b B}\right) \hat{\partial}_{-} X^{b} \hat{\partial}_{+} X^{B}=0 & \text { for } \mathrm{i}=\mathrm{a}
\end{array}
$$

A local solution for the mixed part of the metric is $G_{a B}=\partial_{a} \partial_{B} f$ where $f$ is an arbitrary function of $X^{A}$ and $X^{a}$. The background allowing both movers is then

$$
\begin{array}{r}
G_{A B}=G_{A B}\left(X^{A}\right), \quad G_{a b}=G_{a b}\left(X^{a}\right) \\
G_{a B}=\partial_{a} \partial_{B} f\left(X^{A}, X^{a}\right) .
\end{array}
$$

By substituting (16) in the equations of motion one does indeed see that this is the most general class of metric which allows the presence of both movers. Nevertheless, for an arbitrary metric $G_{i j}$, only the solution with one of the movers present is allowed. For metrics of the form (7) the chiral solution is also unique. This stems from the fact that an arbitrary background of type (7) is more general than an arbitrary background of type (12). 
Now we turn to the study of the stability of the solution. Since our solution can be regarded as, say, a right-mover closed string with its center-of-mass at rest in a comoving frame we have to look for small perturbations with can grow up with time $\tau$ or small perturbations which can change the chirality of the string. The question of stability can then be posed as a problem of finding the eigenvalues $w$ for a perturbation $f(\sigma)$ with small coefficient $\lambda$,

$$
X^{i}(\sigma, \tau)=X^{i}(\sigma-\tau)+\lambda f_{w}^{i}(\sigma) e^{-i w \tau}
$$

As usual, real eigenvalues indicates the stability of the solution. By substituting it into the equations of motion and keeping only the linear terms it can then be easily seen that, due to the dependence on $\tau$ of the Christoffel symbol (and coordinate $X^{j}$ ),

$f^{\prime \prime i}(\sigma)+w^{2} f^{i}(\sigma)=-2\left[\Gamma_{j k}^{i} \dot{X}^{j}\right](\sigma, \tau)\left[i w f^{k}(\sigma)-f^{\prime k}(\sigma)\right]$

the eigenvalue equations decouple and we get $f(\sigma)=e^{i w \sigma}$ with $w$ real. Then the perturbation does not change the character of the solution (it remains a right- mover) and we conclude that the solution is stable under small perturbations.

Besides having zero action, which means a large contribution to the path integral, another important feature of the chiral solution in curved space is its solitonic character. The modes of the string are not affected by the presence of the background, a behaviour that resembles solitons. The same occurs with the winding mode, which, in addition, has a topological character. On the other hand, the Hamiltonian

$$
H=\int d \sigma G_{i j} \dot{X}^{i} \dot{X}^{j}=\int d \sigma G_{i j} X^{\prime i} X^{\prime j}
$$

does depend on the metric. This can be interpreted even if the string is understood as being in a comoving frame, since it is an extended object and its energy is not localized.

As in flat space-time [13] the string winding mode has a solitonic character. Since we have a topological conserved current $J_{\alpha}^{i}=(2 \pi) \epsilon_{\alpha \beta} \partial^{\beta} X^{i}$, there is a conserved charge for a solitonic solution of the form $X_{\text {sol }}^{i}(\sigma-\tau)=2 w^{i}(\sigma-\tau)$, where $w^{i}$ is the winding number. 
A formal expression for the quantum soliton operator which creates a soliton (of winding number $w^{i}$ ) out of the vacuum is given by

$$
T(w)=\exp \left[-\frac{i}{\pi} \int d \sigma^{-} X_{s o l}^{i}(\sigma-\tau) P_{i}(\sigma-\tau)\right]
$$

where $P_{i}$ is the momentum associated to $X^{i}$, i.e, $P_{i}=G_{i j} \hat{\partial}_{-} X^{j}$. This operator satisfies the usual commutation rule with the charge operator $\hat{W}^{i}=(2 \pi)^{-1} \int d \sigma^{-} \hat{\partial}_{-} X^{i}$, that is,

$$
\left[\hat{W}^{i}, T(w)\right]=w^{i} T(w)
$$

We conclude with some comments and remarks. As far as the properties of the string solution are concerned, only local properties of the space-time, like its curvature, are relevant. Topological properties such as a possible nonsimply connected structure or compactedness of the background, are not relevant to select one of the movers. The later properties may contribute to a nontrivial fundamental homotopy group which in turn determines the presence of winding modes only. In connection with this, other backgrounds such as the antisymmetric tensor can be considered. It will contribute with a torsion term to the equations of motion.

The solution allowing both movers eq. (16) could be turned into a Kähler metric if $G_{A B}=G_{a b}=0$ and if a complex structure is properly introduced after Euclideanization. The left- or right-movers turn into holomorphic or antiholomorphic functions and one may interpret them as sigma-model instantons defined on a Kähler manifold [14]. However this interpretation is not valid since our base space is compact (in the world sheet coordinate $\sigma$ ) and it is not possible, by joining a point at infinity to define the second homotopy group needed for the characterisation of instantons.

Besides the static backgrounds which allows left- and/or right-movers, we also found from eqs. (6a), (6b) and (6a) a time-dependent solution

$$
\begin{aligned}
G_{+-} & =\partial_{-} h \\
G_{-i} & =\left[a_{i} X^{+}+g_{i}\left(X^{i}\right)\right] G_{+-}
\end{aligned}
$$




$$
\begin{aligned}
G_{+i} & =a_{i} h+\partial_{i} h \\
G_{i j} & =\frac{G_{+(i} G_{-j)}}{G_{+-}},
\end{aligned}
$$

where the $a_{i}$ are constants, $g_{i}=g_{i}\left(X^{i}\right)$ and $h=h\left(X^{-}, X^{i}\right)$. As pointed out above such a time-dependent solution should not be allowed. In fact, it is possible to show that the metric () is singular. Singular metrics are allowed in gauge theories for gravitation but their role is not understood [15]. Moreover it should be noticed that such a metric admits black hole solutions since, if $G_{+-}$vanishes in some point, then $G_{i j}$ becomes singular (and vice-versa).

Other background fields may be considered. The antisymmetric tensor, for instance, contributes to the equations of motion with a torsion which is the antisymmetric part of the Christoffel symbol. This generalized equation still allows the chiral solution.

\section{ACKNOWLEDGMENTS}

OAM would like to thank the support received at the Instituto de Física of the Universidade de São Paulo, and CAPES for financial support. The work of VOR is partially supported by CNPq. 


\section{REFERENCES}

* $\quad$ E-Mail address: MATTOS@USPIF.IF.USP.BR

$\dagger \quad$ E-Mail address: VRIVELLES@USPIF.IF.USP.BR

[1] C. G. Callan, D. Friedan, E. J. Martinec, and M. J. Perry, Nucl. Phys. B262, 593 (1985).

[2] G. T. Horowitz and A. R. Steif, Phys. Rev. D 42, 1950 (1990).

[3] D. Amati and C. Klimčík, Phys. Lett. B210, 92 (1988); H. J. De Vega and N. Sanchez, Nucl. Phys. B317, 706 (1989).

[4] E. Witten, Commun. Math. Phys. 92, 455 (1984); E. Bergshoeff, S. Randjbar-Daemi, A. Salam, H. Sarmadi, and E. Sezgin, Nucl. Phys. B269, 77 (1986).

[5] A. A. Tseytlin, "A Class of Finite Two-Dimensional Sigma Models and String Vacua", Preprint DAMPT-92-26, 1992.

[6] For a review see: A. M. Perelomov, Phys. Reports 146, 135 (1987).

[7] R. Floreanini and R. Jackiw, Phys. Rev. Lett. 59, 1873 (1987); L. Faddeev and R. Jackiw, ibid. 60, 1692 (1988); M. E. V. Costa and H. O. Girotti, ibid. 60, 1771 (1988); H. O. Girotti, M. Gomes, V. Kurack, V. O. Rivelles and A. J. Silva, ibid. 60, 1913 (1988); H. O. Girotti, M. Gomes, V. O. Rivelles and A. J. Silva, Phys. Rev. D 39, 3792 (1989).

[8] D. J. Gross, J. A. Harvey, E. Martinec, and R. Rhom, Nucl. Phys. B256, 253 (1985).

[9] W. Siegel, Phys. Lett. B206, 631 (1988).

[10] See for instance, M. B. Green, J. H. Schwarz, and E. Witten, Supersting Theory, (Cambridge University,Cambridge,1987), Vol. 1.

[11] A space $M^{2} \oplus K$, with $M^{2}$ being the 2-dimensional Minkowski space and $\mathrm{K}$ some 
curved space (not necessarily compact), enables through the LCG to determine the $X^{-}$coordinate algebraically in terms of transverse coordinates. With our choice for the metric one can still determine $X^{-}$by solving a differential equation.

[12] S. Weinberg, Gravitation and Cosmology (John Wiley \& Sons,New York,1972).

[13] Z. F. Ezawa, S. Nakamura, and A. Tezuka, Phys. Rev. D 34, 3805 (1986).

[14] A. M. Perelomov, Commun. Math. Phys. 63, 237 (1978).

[15] E. Witten, Nucl. Phys. B311, 46 (1988). 